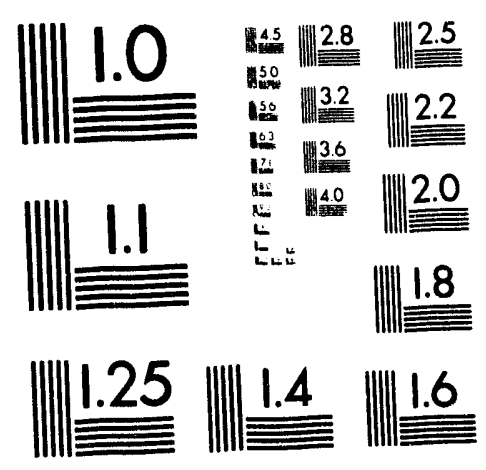



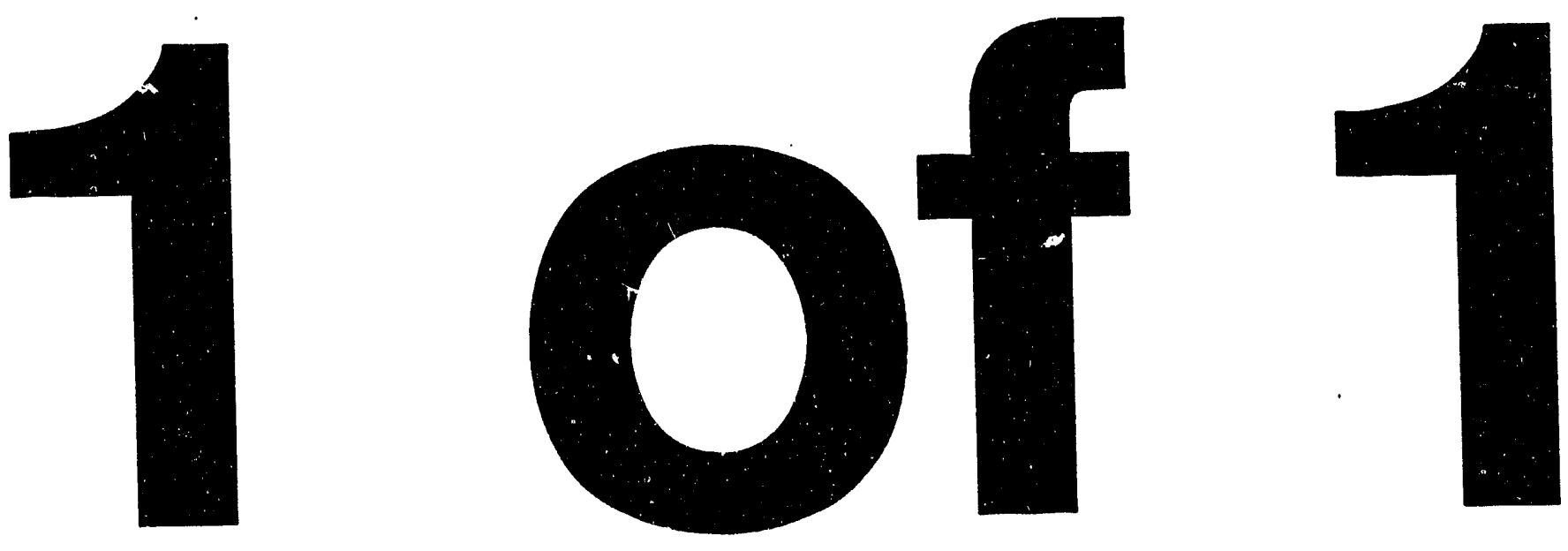
UCRL-LR-114552

Distribution Category UC-700

\title{
Legacy of the X-ray Laser Program
}

\author{
Joseph Nilsen
}

Manuscript date: August 6, 1993

\section{LAWRENCE LIVERMORE NATIONAL LABORATORY University of California $\cdot$ Livermore, California $\cdot 94551$}

Available to DOE and DOE contractors from the Office of Scientific and Technical Information

P.O. Box 62, Oak Ridge, TN 37831 Prices available from (615) 576-8601, FTS 626-8401

Available from: National Technical Information Service $\bullet$ U.S. Department of Commerce 5285 Port Royal Road • Springfield, VA $22161 \cdot$ A04 $\bullet$ (Microfiche A01) 


\section{Legacy of the X-Ray Laser Program Joseph Nilsen}

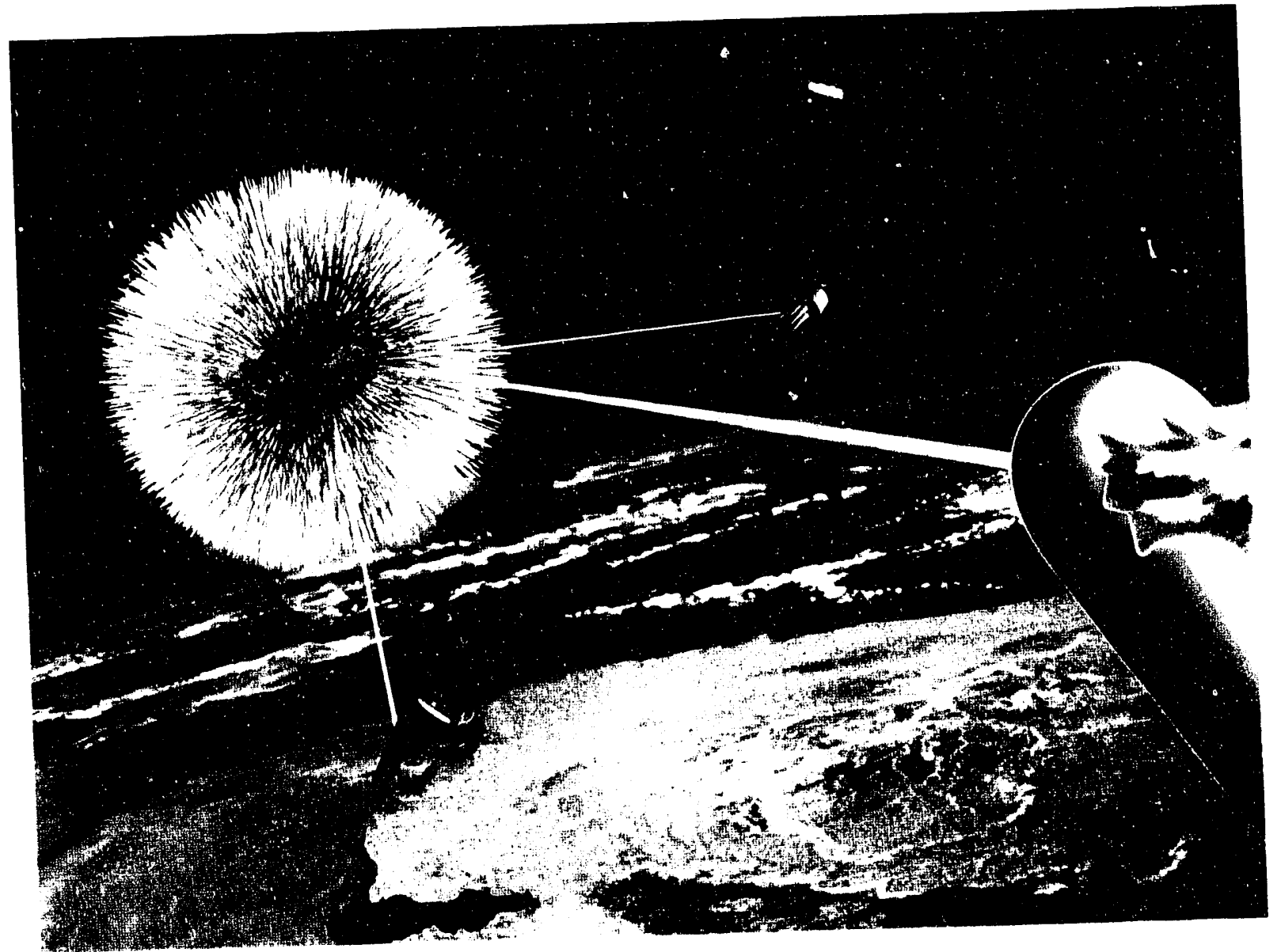

The X-Ray Laser Program has evolved from a design effort focusing on developing a Strategic Defense Initiative zeapon that protects against Soviet ICBMs to a scientific project that is producing new technologies for industrial and medical research.

\section{Introduction}

Interest in producing shorter wavelength lasers which reached into the $X$-ray region' began soom after the operation of the first ruby laser in lyo(l). With the advent of the large lasers for inertial confinement fusion (ICF) in the early 1970 's, propouals for investigating $X$-ray lasers were made by several groups ${ }^{2,3}$ including those in the Soviet Union and at Lawrence Livermore National Laboratory (LLNL). Scientists at LLNL. become intrigued by the possibility of producing a nuclear pumped $\dot{X}$-ray laser that could be used as an anti-ballistic missile (ABM) system. At the same time a large number of articles in the literature suggested that the Soviet Union was 
pursuing such a system. ${ }^{+7}$ As a conseyuence, work began at LLNL to explore such systems. By the time Ronald Reagan was elected President in 198(), a significant study of the nuclear pumped $X$-ray laser was well under way. Edward Teller emerged as a champion of the X-ray laser for use as a potential ABM system and discussed the idea with the new President, reasoning that there was potential for a real breakthrough in strategic defense against intercontinental ballistic missiles (ICBMs). For the first time there appeared to be a real opportunity to change the current policy of mutually assured destruction (MAD) and defend the USA against Soviet attack. With the advice of Dr. Teller and others, President Reagan gave his so-called "Star Wars" speech on March 23, 1983, in which he outlined his vision for a shield over the USA that would finally end the MAD policy. Thus began the Strategic Defense Initiative (SDI) that funded the $X$-ray laser effort as well as numerous other research activities such as the development of free electron lasers (FELs), chemical lasers, and Brilliant Pebbles.

Over the last ten years the world changed dramatically. The Berlin Wall has fallen, Germany has been reunited, and the Soviet Union has collapsed. Because of this new world order and the technical difficulties in developing the $X$-ray laser as an $A B M$ system, the nuclear pumped $X$-ray laser effort has been eliminated. However, many great technical and physics achievements resulted from the X-Ray Laser Program. While the great technical successes and failures of the $X$-ray laser itself cannot be discussed, this article presents the many significant achievements made as part of the X-ray laser effort that are now being used for other applications at LLNL.

\section{Diagnostics}

In the 1970's most measurement systems for nuclear tests relied on coaxial cables connected to single $X$-ray diodes. As electronics technologies advanced, fiber optics, streak cameras, multiplexing, and other new technologies began to be used. Then, with the advent of the X-ray laser effort, suddenly vast quantities of high resolution data were needed that could not be gathered using conventional techniques. This need for more detailed data pushed the immense growth made in the development of advanced electrooptics devices used in nuclear tests.

\section{Transmission Crystal Spectrometer}

A good example of the tremendous progress in measurement systems technologies is the transmission crystal spectrometer (TRACS). ${ }^{8}$ Before the development of this spectrometer, hard $X$-rays were measured in the nuclear environment using absorption-edge filters and fluorescers to define handpass regions of the $X$-ray spectrum. This diagnostic approach could accommodate very few data channels, and therefore the amount and type of data that could be gathered was severely restricted. For example, a standard instrument like the SPECTEX had 10 spectral-energy channels. The TRACS solved the crystal spectroscopy problem by providing a way to measure short wavelength $X$-rays (i.e., hard $X$-rays) by using a crystal with the planes perpendicular to the crystal surface, as shown in Fig. 1, and then measuring the transmission spectroscopy of the $X$-rays. The $X$-rays still have small Bragog angles with respect to the Bragg planes, but now they strike the crystal at nearnormal incidence. Using the TRACS one can now have 1000 channels of time-resolved data covering the same spectral range as the SPECTEX, an increase of two orders of magnitude in resolution.

\section{High-Precision Spatially Resolved X-Ray Spectrometer}

Measuring the electron temperature of a plasma is very important to our understanding of the dynamics of nuclear-driven plasmas. To gather this type of information a high-precision spatially resolved $X$-ray spectrometer was developed that measures the free-bound continuum emission from radiative recombination. ${ }^{4}$ In one particular experiment, four samples were imaged simultaneously with subnanosecond time resolution. This required very stringent pointing tolerances that were not previously possible. A 20 - $\mu$ rad pointing accuracy was achieved, which was 5 times better

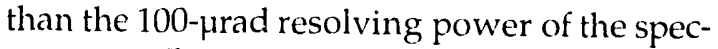
trometer. The electron temperatures of both the Kand $L$-shell nuclear-driven plasmas were determined to an accuracy of better than $10 \%$.

\section{Fast X-Ray Detectors}

A new generation of fast $X$-ray detectors was developed to meet the challenges of diagnosing plasmas in a nuclear environment. ${ }^{10}$ The new 
detectors are based on microchannel plate-intensified detectors developed for diagnosing laboratory $X$-ray lasers. These detectors have a 400 -ps time resolution and convert the X-ray signal into a 1000times-brighter optical signal. This is a million-fold conversion efficiency enhancement when compared with the passive phosphors and scintillators used previously. Figure 2 shows a schematic of the detector with the photocathode that converts $X$-rays to photoelectrons, the microchannel plate (MCP) that amplifies the photoelectrons, the electrostatic focusing electrode that compresses the electron current in one dimension, the fast phosphor that converts the electron's kinetic energy to light, and the fiber-optic face plate that transmits the light out of the vacuum system.
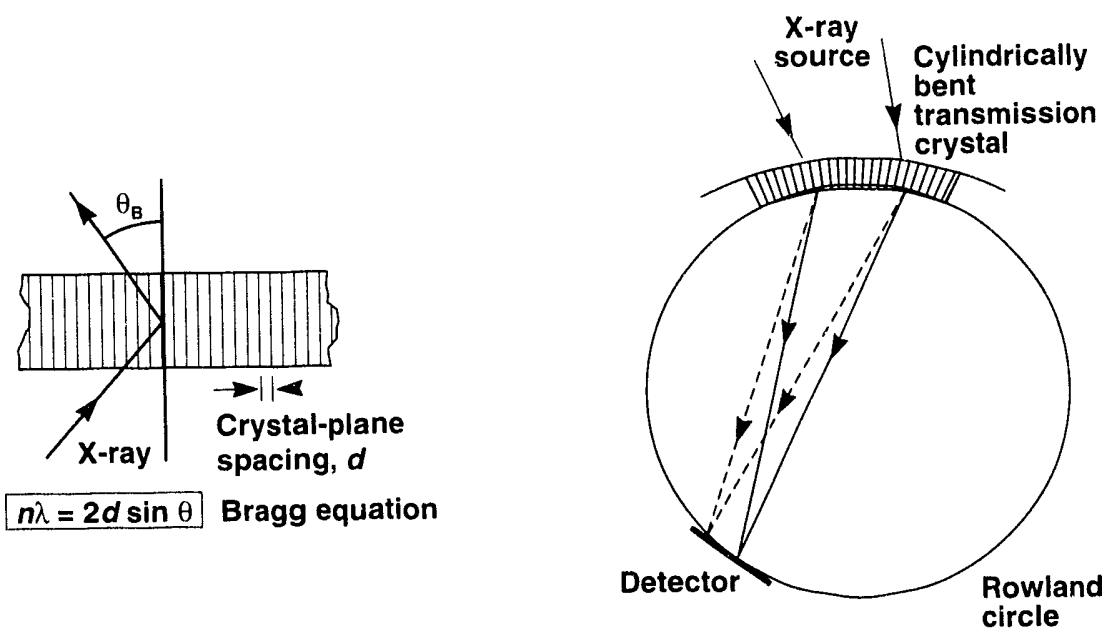

Figure 1. Transmission crystal spectrometer (TRACS) for measuring hard X-rays underground. The TRACS uses a crystal with the planes perpendicular to the crystal surface, and measures the transmission spectroscopy of the X-rays.

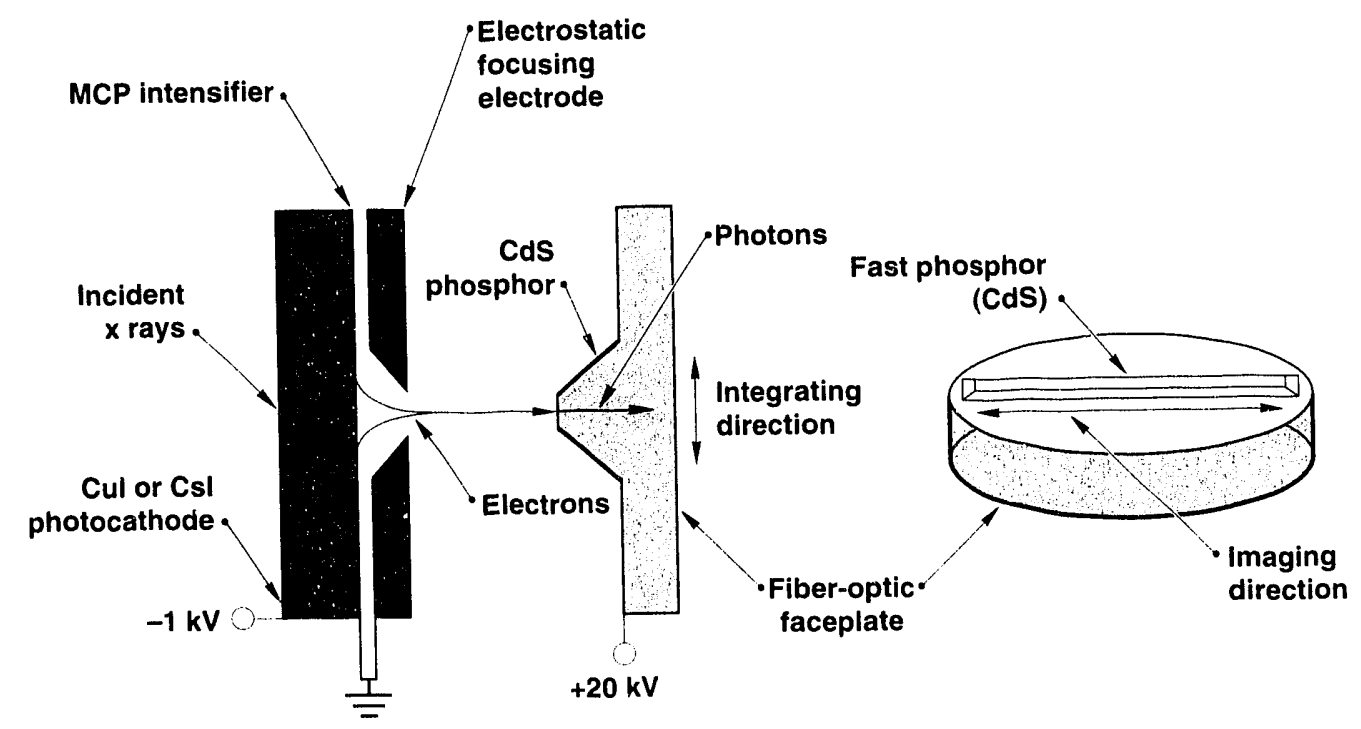

Figure 2. Time-resolving intensified detectors for low-level X-ray detection. The photocathode converts $\mathrm{X}$-rays to photoelectrons and the microchannel plate (MCP) intensifier amplifies the photoelectrons. The electrostatic focusing electrode compresses the electron current in one dimension, and the CdS phosphor converts the electron's kinetic energy to light. Finally, the fiber-optic faceplate transmits the light out of the vacuum system. 


\section{Laboratory X-Ray Laser}

As part of the undertaking aimed at developing an understanding of $X$-ray laser physics and developing laser-physics computation codes, the laboratory $X$-ray laser effort began. In the beginning it used the Novette laser, which was a prototype for the Nova laser. In 1984 the first laboratory $X$-ray laser was demonstrated at LLNL; it lased primarily at 206 and $209 \AA$ using a plasma of selenium, which was stripped of 24 electrons to the neon-like state. ${ }^{11,12}$ Figure 3a shows the on-axis spectrum from a $1.8-\mathrm{cm}$-long selenium $\mathrm{X}$-ray laser. The spectrum is dominated by five laser lines, the weakest of which completely dwarfs the other strong emission lines from sodium-like selenium (i.e., the $201 \AA$ line). Figure $3 \mathrm{~b}$ shows the time history of the $206 \AA$ laser line, which lasts about 200 ps as compared with the 500 ps Nova heating pulse. The Defense Sciences Department decided to fund a second target chamber on the Nova laser that would allow two beams for X-ray laser experiments. This two-beam Nova facility has now been in use for 7 years and has demonstrated many new $X$-ray lasers whose wavelengths range from $326 \AA$ in neon-like titanium to $35 \AA$ in nickel-like gold. ${ }^{13}$

\section{X-Ray Imaging}

One of the potential uses of laboratory X-ray lasers is in imaging. $X$-ray lasers are now being coupled with $X$-ray microscopes to image biological samples. X-ray microscopes that use $X$-ray lasers for their light source can potentially image hydrated organisms in physiolngically normal conditions-"live specimens"-revealing their living structure. This technology is paving the way to eventually creating three-dimensional (3-D) holograms of living organisms. As the first steps towards making living biological images, an X-ray microscope has been constructed that uses a $44.83 \AA$ nickel-like tantalum X-ray laser as the light source, a spherical multilayer mirror for a condenser, and a zone plate for the lens that projects the image onto the microchannel plate detector ${ }^{14}$ (see Fig. 4a). This X-ray microscope has captured images of rat sperm (see Fig. 4b) that show $500 \AA$ features. This technology is being used to look at how DNA is organized inside a sperm cell. ${ }^{15}$

To improve the coherence and power of X-ray lasers, multipass amplification was demonstrated at $206 \AA$ in a neon-like selenium laser by using multilayer mirrors made of 30 molybdenumsilicon pairs with a $111 \AA$ A period. ${ }^{16,17}$ In these early experiments, a flat multilayer mirror was placed $2.75 \mathrm{~cm}$ from the end of the laser medium. Data collected at the right end of the laser (see Fig. 5) showed both the amplified spontaneous emission (ASE) pulse from the $X$-rays that originated at the left end of the laser (these $X$-rays were amplified as they propagated down the length of the laser), and a second pulse, created by $X$-rays starting at the right end. This second pulse was observed after it propagated down the laser, was reflected by the
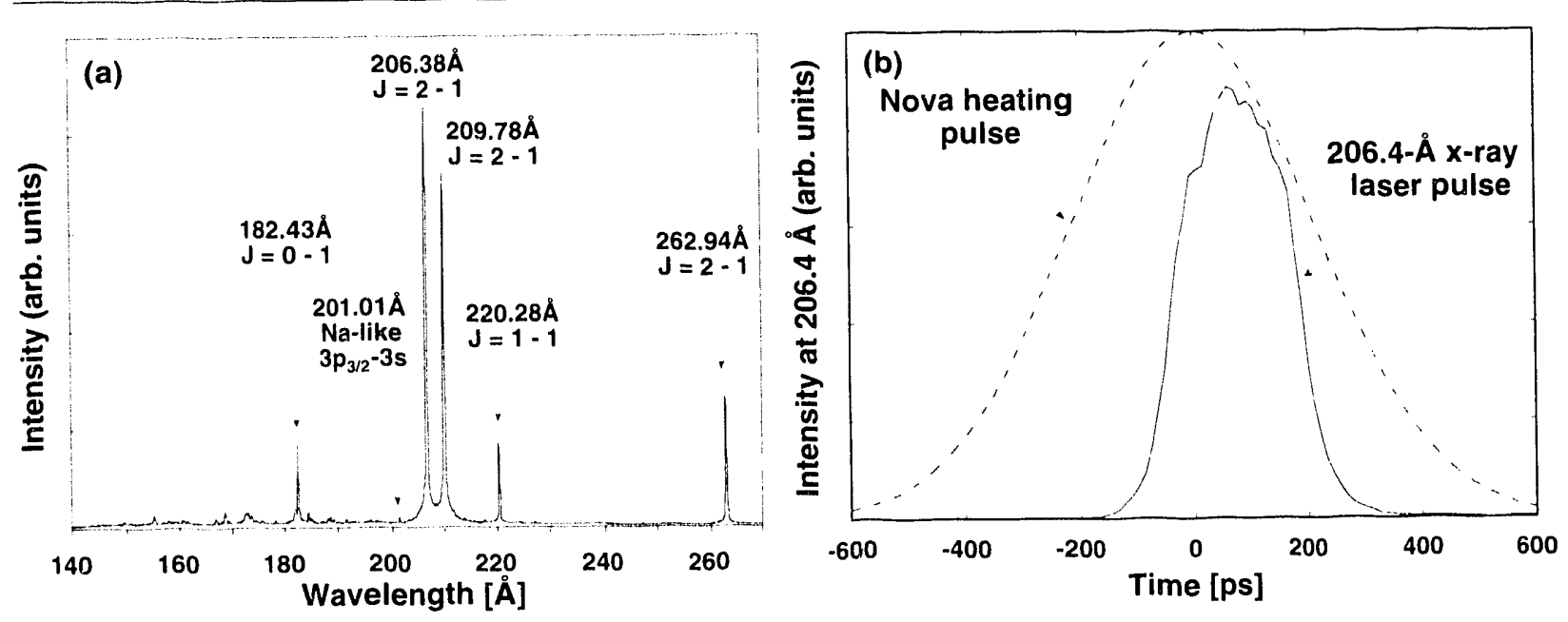

Figure 3. (a) Typical on-axis spectrum from a 1.8-cm long neon-like selenium $X$-ray laser. (b) Time history of the $206.4 \AA$ laser line as detected on a sireaked spectrometer. The dashed curve represents the time history of the 500-ps Gaussian Nova heating pulse. 
(a)

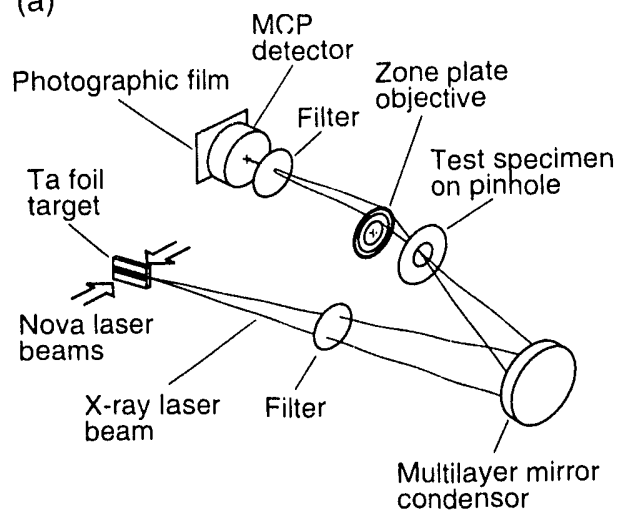

$10 \mu$

(b)

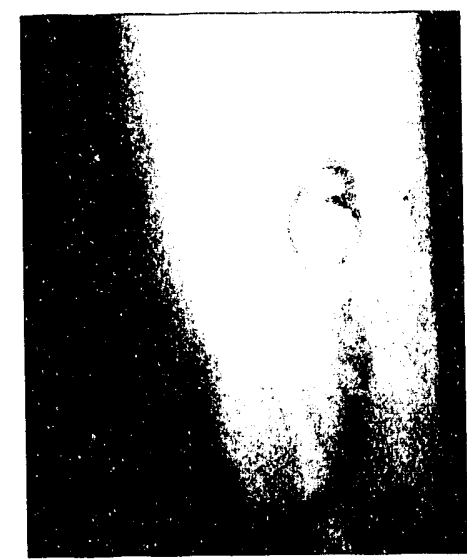

Figure 4. (a) Diagram of the X-ray microscope. The light source is a nickel-like tantalum $X$-ray laser. The $X$-ray laser beam is condensed using a spherical multilayer mirror, and a zone plate projects the image onto the microchannel plate detector. (b) Image of rat sperm captured using the X-ray microscope. This image shows $500 \AA$ features.

(a)

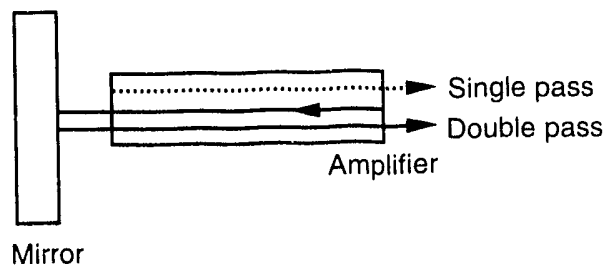

(b)

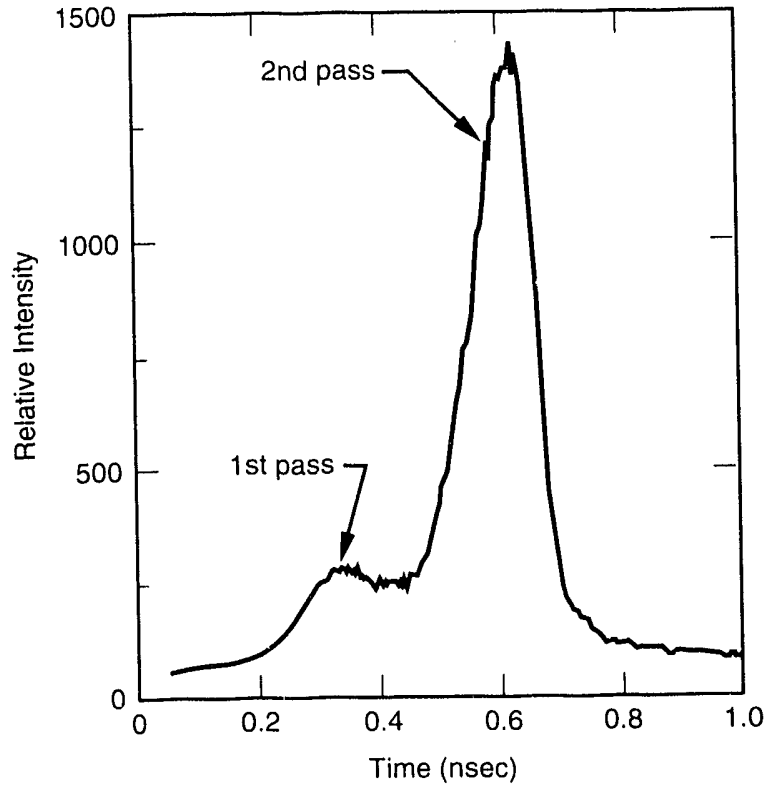

Figure 5. (a) In the experiment demonstrating multipass amplification of the X-ray laser, the $X$-rays in the first pulse originated at the left end of the laser and were amplified as they propagated down the length of the laser. The $X$-rays in the second pulse originated at the right end of the laser, were reflected by the mirror, and then were reamplified during their second pass. (b) Measurements show that multipass amplification of the X-ray laser increased coherence and power of the laser-the double-pass amplified signal was seven times more intense than a single-pass signal. 
mirror, and then was reamplified during its second pass through the gain medium (see Fig. 5a). This double-pass amplified signal was seven times more intense (see Fig. 5b) than the single-pass ASE. This experiment demonstrated the first step toward making an $X$-ray laser cavity, which offers the potential for producing the highly coherent output required to do holography.

These multilayer mirrors have also been very important in developing new diagnostics in the soft $X$-ray regime where crystal spacings are too small to allow measurements to be made. A variation of the multilayer technology is Fresnel zone plates, which are essentially multilayer mirrors rotated 90 degrees.

A tabletop X-ray microscope which achieves $10 \mu$ resolution has been built using $8 \mathrm{keV}$ Fresnel zone plates. This microscope creates images of a sample by scanning it with a focused $X$-ray spot that is produced using a phase-modulating zone plate and a $\mathrm{Cu} \mathrm{K}-\alpha$. source. The picture of the ant shown in Fig. 6 is an example of an image made using this microscope. ${ }^{18}$

\section{Codes}

The design of the laboratory X-ray laser required the ability to make very complex atomic models that could be used for solving the plasma kinetics. Several different codes had to be developed to do this. One was the YODA code. ${ }^{14}$ It was developed to calculate detailed energy levels, oscillator strengths, collision rates, and auger rates. The ADAM code filled in the hydrogenic data for levels and sequences that were not described in detail. To help with the detailed atomic physics calculations, other codes such as the MCDF code ${ }^{2(1)}$ of Grant and the HULLAC code $21-2.3$ were brought to LLNL and incorporated in newer model-building codes.

For designing the neon-like selenium laser, LASNEX was used to calculate the temperatures and hydrodynamics of the optical laser-selenium foil interaction. The output of LASNEX, in the form of electron and ion temperatures and densities versus space and time, was used as input to XRASER, which was used to calculate level populations and gain. ${ }^{24}$ XRASER also calculated the radiative transfer ${ }^{25}$ in a plasma, accounted for the effects of radiation trapping on the gain, and transferred the pump radiation to the lasing plasma (i.e., for the resonantly photopumped laser scheme).
Recent 3-D calculations ${ }^{2 n}$ have been done using XRASER to model the beam pattern from the nickel-like tantalum laser at $44 \AA$. These calculations are the first to include transverse hydrodynamics, refraction effects, and longitudinal propagation for non-uniform illumination along the laser axis.

\section{New Materials}

One of the exciting developments has been in the area of very low density foams such as silica aerogel, organic aerogels, and safe-emulsion agar gel (SEAgel). Silica aerogel was first prepared in the 1930 's at Stanford University, but it did not

(a)

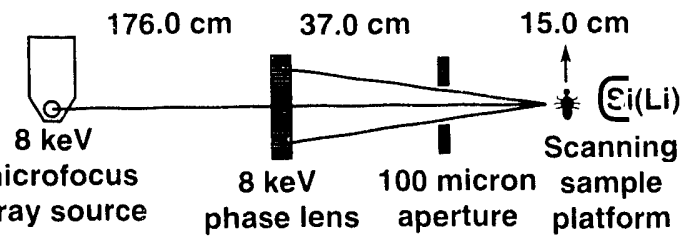

(b)

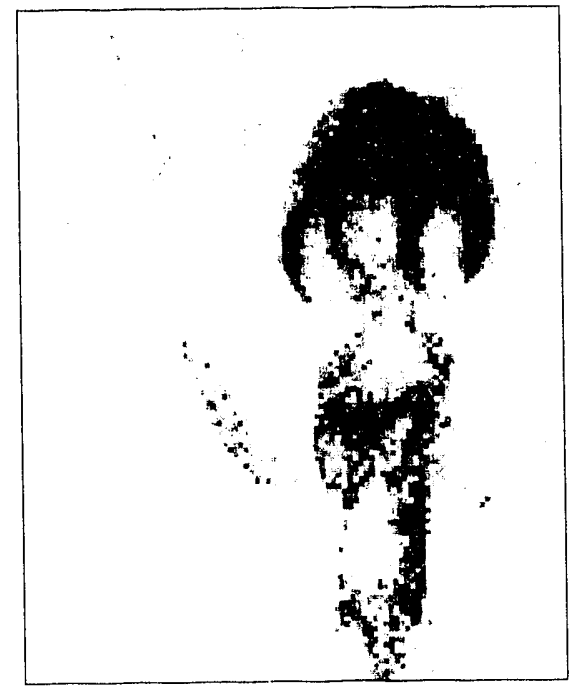

Figure 6. Example of an image made with the scanning $X$-ray microscope, using an ant. (a) Schematic of the $8 \mathrm{keV}$ scanning $X$-ray microscope geometry (not to scale). This tabletop X-ray microscope creates images by scanning a sample with a focused $X$-ray spot that is produced using a phase-modulating zone plate and a copper $K-\alpha$ source. (b) The image of the ant shows features as small as $50 \mu$, with each pixel corresponding to $15 \mu$. 
find a practical use until the 1970's when it was used in nuclear particle detectors. At LI NL in the 1980's, aerogel technology took a leap forward with the creation of super-light silica aerogels (densities of $1 \mathrm{mg} / \mathrm{cc}$ ) and the production of organic aerogels. ${ }^{2-24}$

Aerogels are open cell structures with cell sizes less than 500 A. These small cell sizes make the foam transparent to light. They also have outstanding thermal insulating and acciustic properties, making them candidates for use as insulation in windows, refrigerators, and many other applications. A good example of the insulation value of aerogel is shown in Fig. 7, which shows a photograph of a person heating a piece of aerogel foam with a torch while holding it in his hand. In another new application, aerogels are being used to catch micrometeoroids (cosmic dust) in space. Potentially, organic aerogels may find a variety of uses such as gas filters in chemical processing, catalyst beds for the petroleum industry, and thermal insulators for cryogenic applications.

A very low density organic based foam is SEAgel. SEAgel is an all-natural material made from agar, a component of red algae used to thicken ice cream and other foods. Because SEAgel is safe enough to eat, its potential applications range from insulation, to packaging material, to slow-release capsules for medicines. SEAgel is made by dissolving agar in water, then adding an organic solvent and emulsifier that disperses the agar evenly throughout the liquid. Next, it is freeze-dried to extract the water and solvents. It can be made with densities from 1 to 300 milligrams per cubic centimeter and with cell sizes from 2 to 3 microns. A very useful application of SEAgel is the replacement of balsa wood, which is becoming hard to find because it is widely used to insulate supertankers and provide sound damping in high-speed trains. ${ }^{3(1)}$ Figure 8 graphically illustrates the very low density of SEAgel; a piece of SEAgel is shown floating on soap bubbles.

SEAgel is being used as laser targets for X-ray laser experiments on Nova because it can be doped with other materials such as selenium. At the present time, we are trying to fabricate an X-ray laser target with a density that is less than the critical density $\left(\mathrm{N}_{\mathrm{c}}=4 \times 10^{21}\right.$ electron $/ \mathrm{cc}$ for $0.53 \mu$ light) to avoid the violent hydrodynamics that take nlace when a solid density target explodes before It reaches the density required for lasing. Our goal is to achieve a more uniform plasma, which will improve the quality of the X-ray laser beam.

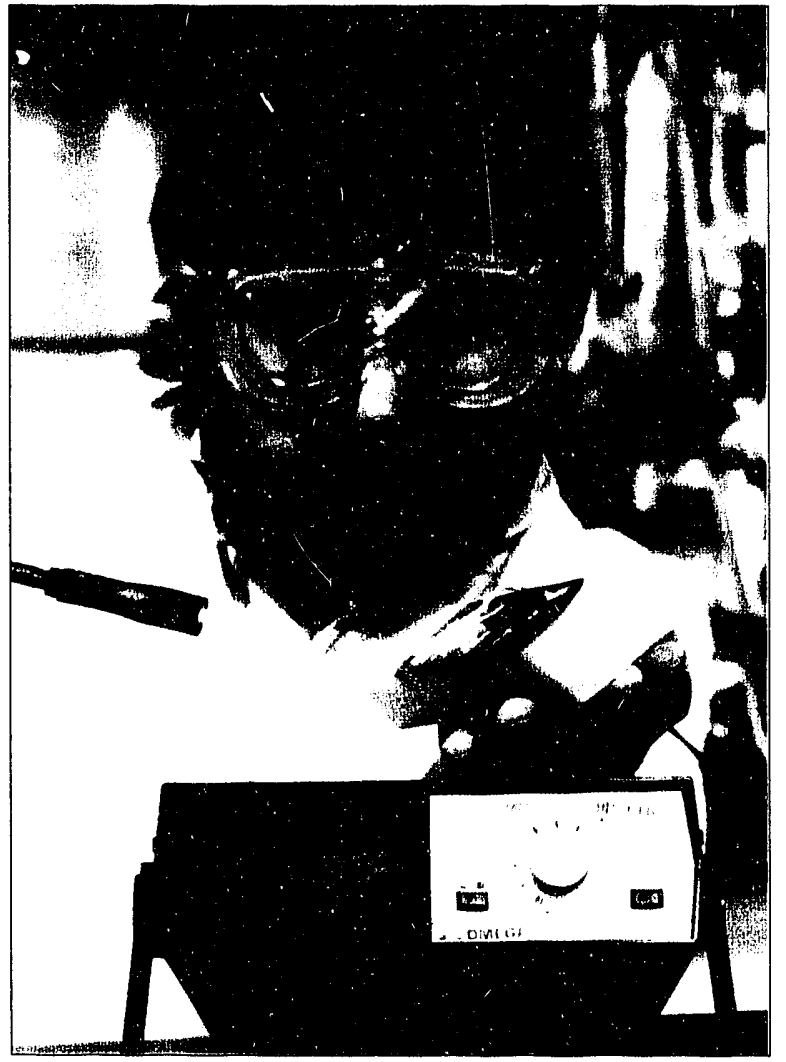

Figure 7. Tom Tillotson, a chemist at Lawrence Livermore National Laboratory, applies a propane torch to the top of an inch-thick silica aerogel brick, while his hand below remains totally unaffected by the heat.

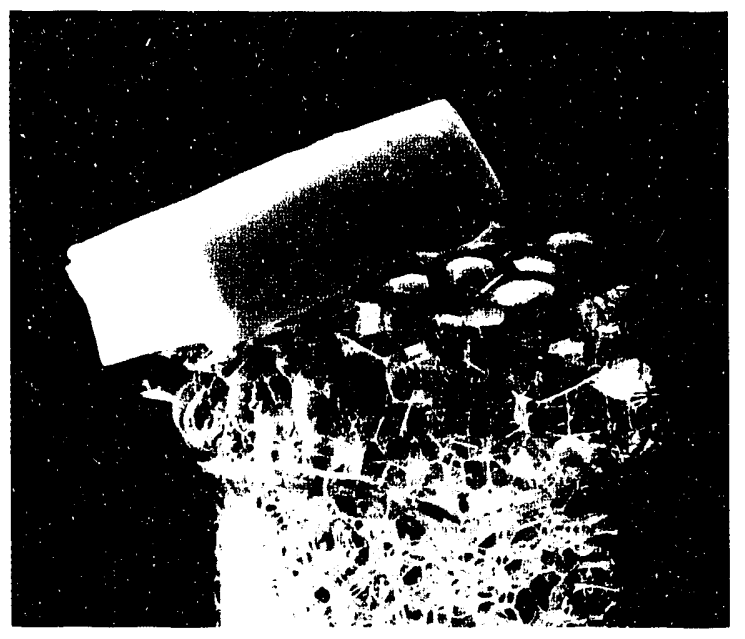

Figure 8. A piece of SEAgel (Safe Emulsion Agar gel) has a density so low it floats on soap bubbles in a beaker. SEAgel is an all-natural material made from agar, a substance used to thicken foods. 


\section{Material Analysis}

Building experiments that were to be included with nuclear tests required very strict manufacturing tolerances. These tolerances were met by developing many new characterization techniques capable of handling many thousands of new parts a year.

As an outgrowth of our radiography activities, a superior mammographic technicye is being developed that potentially may increase the early detection of breast cancer, save lives, anc. reduce health care costs. ${ }^{31}$ It is estimated that hreast cancer will strike approximately $10 \%$ of women in the USA during their lifetime, and early detection is the best defense against the disease. Currently, mammograms are used to detect the micro-calcifications that often accompany the beginning stages of breast cancer. At present, analyzing mammograms requires a highly trained individual and is subjective, with the early microcalcifications often missed. The quantitative radiography techniques developed at LLNL offer the promise to make the computer identification of micro-calcifications faster and more reliable than unassisted human interpretation. Figure 9 shows an example of a digitized X-ray mammogram. The calcifications identified by the computer program are highlighted with circles.

Other applications of the radiographic meth ds have found a use in the biomedical industry. Using a diagnostic tec'. inique developed to find tiny flaws in SDI components, scientists have recently been able to identify a potentially fatal flaw in a sar.pie of artificial-valve material. ${ }^{32}$ With the current method, a sample of heart valve material is placed in a vacuum chamber and bombarded with a $10 \mathrm{MeV}$ proton beam. The sample is then analyzed by a detector that me?sur 's the beam's energy. Based on the energy loss iit ine beam, the thickness of the material is determined. Recent experiments were able to detect a minute scuff in the core of a sample of carbonbased heart valve material. There are currently 1.5 million people with artificial heart valves. Small defects can have fatal consequences. The inability to identify defective material has already driven one manufacturer out of the market.

Three-dimensional X-ray computer tomography, which was developed to inspect weapons parts, is now being used in civilian and medical applications. The basic technique illuminates a sample with a collimated monochromatic X-ray source to create a two-dimensional image. By rotating the samples in small angular increments through 180 degrees. enough data is obtained to reconstruct a 3-dimensional image of the sample with 2-3 $\mu$ resolution using $X$-rays in the 5-35 $\mathrm{keV}$ range. This technology is similar to the CAT scan done at the hospital, but has a much higher resolution. This technology is being used to look for pores in ceramics composites, to identify potential fatigue areas in tensile loading of aircraft wings, and to study demineralization of dentin, which leads to tooth decay. ${ }^{33}$

A continuum $X$-ray gauging technique ${ }^{34}$ has been developed for nondestructive elemental analysis of materials with known constituents. (a) Digitized mammogram

Figure 9. (a) A digitized mammogram. For reference, part of the pectoral muscle is visible at top left. (b) The most suspicious microcalcifications in the area bordered by a rectangle in (a) can be highlight d with circles. A mammographer can focus on the circles in the context of the overall breast structure.

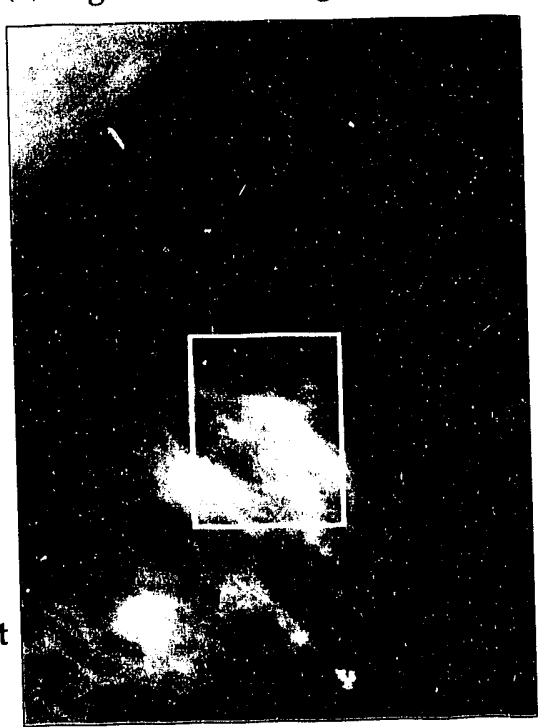

(b) Suspicious microcalcifications

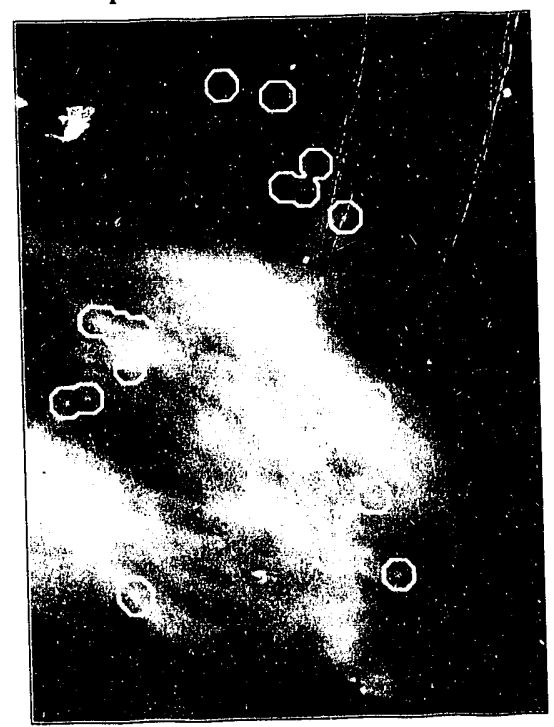


For many applications the quantitative distribution of the components must be known. By measuring the transmission of photons at different energies, the abundance of different components can be mapped.

\section{Electron-Beam Ion Trap}

The X-ray laser program required a new level of understanding and measurements of the atomic physics of highly charged ions. The electron-beam ion trap (EBIT) was developed and built at LLNL ${ }^{35}$ as part of the effort to understand and measure the cross sections and wavelengths of highly charged ions. Currently this trap is capable of producing ions up to neon-like uranium, which requires removing 82 electrons from the uranium atom. One of the first uses of EBIT was measuring the monopole, collisional, excitation cross section for neon-like barium $(Z=56)$ at several electron energies. Since the neon-like selenium $(Z=34)$ laser was predicted to lase based on the same kind of monopole collisional excitation, the EBIT measurements of barium, while at a much higher $\mathrm{Z}$ than the selenium, tended to support the theoretical calculations. ${ }^{36}$

Recently, EBIT has been used to measure another kind of cross section, the dielectronic recombination cross sections for neon-like xenon $(\mathrm{Z}=54))^{37}$ The EBIT measurement and theoretical calculations are in good agreement, which is important since dielectronic recombination is an important process in modeling the ionization balance in plasmas of neon- and nickel-like lasers.

One interesting application of EBIT has been to measure resonances between different ions with very high accuracy. ${ }^{38}$ As part of the effort to develop a resonantly photo-pumped X-ray laser, numerous laser schemes have been proposed. Resonances of a few $100 \mathrm{ppm}$ are needed to make the laser work, but calculations are generally only able to predict a resonance to $1000 \mathrm{ppm}$, and in the past most measurements of this type also had uncertainties of $1000 \mathrm{ppm}$. One proposed laser scheme uses a helium-alpha line of argon to resonantly photo-pump the $2 p \rightarrow 5 d$ transition in neon-like yttrium and enhance the lasing on the $3 p \rightarrow 3$ s lasing line at $155 \AA$. (see Fig. 10a). ${ }^{39}$ Figure $10 \mathrm{~b}$ shows that EBIT measures the helium-alpha line at $3.94911 \AA$ and the neon-like yttrium $2 \mathrm{p} \rightarrow 5 \mathrm{~d}$ line at $3.94849 \AA$. This shows that the resonance is good to $157 \mathrm{ppm}$ and is measured with an accuracy of $28 \mathrm{ppm}$. This is an excellent resonance measured with incredible accuracy.

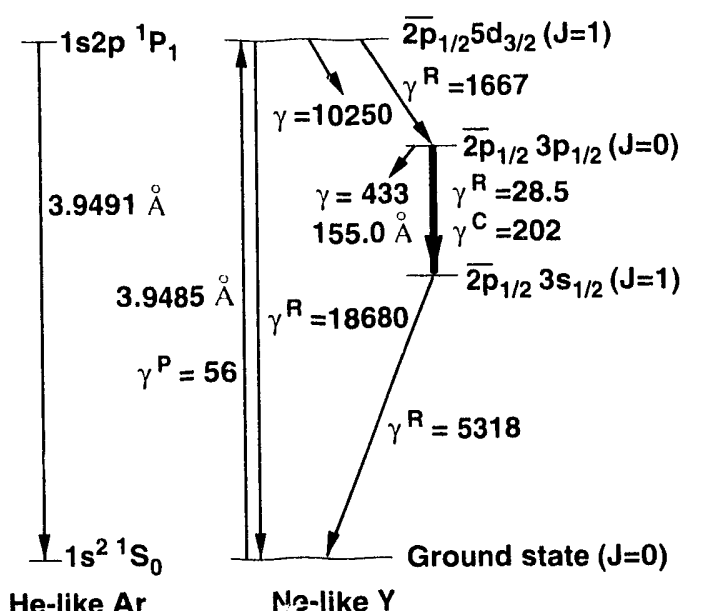

Figure 10 (a). Proposed laser scheme uses a helium-alpha line of argon to resonantly photopump the $2 p \rightarrow 5 d$ transition in neon-like yttrium and enhance the lasing on the $3 p \rightarrow 3$ s lasing line at $155 \AA$. The important radiative and collisional rates, $\gamma^{\mathrm{R}}$ and $\gamma^{\mathrm{C}}$, are shown (in inverse nanoseconds).
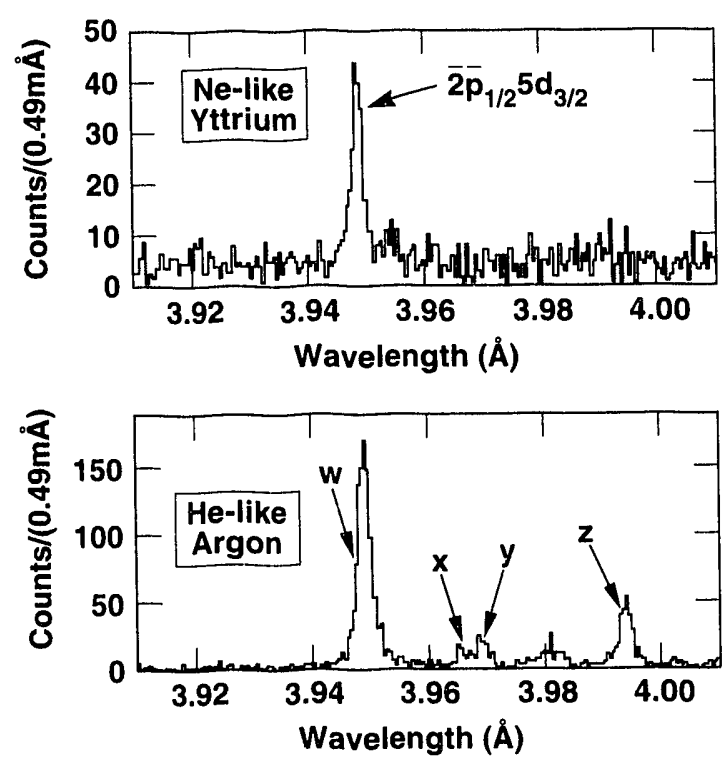

Figure 10 (b). The electron-beam ion trap (EBIT) measures the helium-alpha line, labeled $w$, at 3.94911 $\AA$ and the neon-like yttrium $2 p \rightarrow 5 d$ line at 3.94849 $\AA$. These results show an excellent resonance measured with high accuracy. 
The first observation ${ }^{40}$ of magnetic octupole decay in the X-ray spectra of highly charged jons was made with EBIT. In the low-energy region of the $n=3 \rightarrow n=4$ spectra of nickel-like thorium and uranium, the transitions $3 d^{9} 4 s(J=3) \rightarrow 3 d^{110}$ $(J=0)$ have been measured with a precision of 80 ppm. Observation of forbidden transitions such as these provide fundamental tests of atomic structure theory and kinetics calculations of atomic level populations.

A new EBIT has now been built at LLNL. It is called Super-EBIT because it has electron beam energies up to $200 \mathrm{keV}$ and can strip ions, including uranium, down to the $\mathrm{K}$ shell. One of the newest experiments being undertaken with Super-EBIT is to look for the first observation of bound-state beta decay. It has been predicted that certain nuclei that are stable when they are part of neutral atoms will decay because of bound-state beta decay when the atoms are highly ionized. It is know'n that neutral ${ }^{16} \cdot 3$ holmium decays into ${ }^{16.3}$ dysprosium by electron capture from the $M$ shell or higher. It is therefore expected that the reverse process will happen if all the electrons are stripped off ${ }^{16.3} \mathrm{dysprosium}$ (i.e., ${ }^{16.3} \mathrm{dysprosium}$ will decay to ${ }^{163}$ holmium when a neutron decays into a proton, an antineutrino, and an electron, which is captured directly into the $\mathrm{K}$ or $\mathrm{L}$ shells). The bound-state beta decays for other ions, such as ${ }^{205}$ thallium $\rightarrow{ }^{205}$ lead, are of special interest because it may be used to measure the historical solar neutrino flux. These measurements will be made in the coming year.

\section{Conclusion}

The world has changed dramatically over the last decade and the X-ray laser that Edward Teller so boldly presented to the President is no longer being pursued. But the decade of work on the X-ray laser has produced a rich and important legacy that includes better physics understanding of X-ray lasers; sophisticated computational tools for modeling plasma physics; a laboratory X-ray laser for biological imaging; advanced materials, such as aerogel and SEAgel; new measurement techniques for characterizing materials; and unique, world class facilities like EBIT for performing atomic physics experiments. Over the last decade hundreds of people have participated in this effort and helped to dramatically reshape LLNL. Their achievements have made Lawrence
Livermore National Laboratory a world leader in many fields of science and technology.

\section{Acknowledgments}

The author would like to thank Gus Carlson, Peter Beiersdorfer, Clint Logan, Bob Morrison, Larry Hrubesh, Elaine Chandler, John Kinney, Luiz DaSilva, Brian MacGowan, Mark Eckart, Ken Skulina, Shirley Jennings, and Jane Olivera for their assistance in the preparation of this article. The author would also like to thank Joe Sefcik, who invited him to write this article for inclusion in a future special issue of Energy $\mathcal{E}$ Technology Revieze dedicated to Edward Teller.

\section{Acronyms}

$\begin{array}{ll}\text { ASE } & \text { Amplified spontaneous emission } \\ \text { EBIT } & \text { Electron-beam ion trap } \\ \text { FELs } & \text { Free electron lasers } \\ \text { ICF } & \text { Inertial confinement fusion } \\ \text { keV } & \text { Kilo-electron-volts } \\ \text { LLNL } & \text { Lawrence Livermore National } \\ & \text { Laboratory } \\ \mu r a d & \text { Micro radian } \\ \text { MeV } & \text { Million electron volts } \\ \text { ppm } & \text { Parts per million } \\ \text { ps } & \text { Picosecond } \\ \text { SDI } & \text { Strategic Defense Initiative } \\ \text { SEAgel } & \text { Safe-emulsion agar gel } \\ \text { TRACS } & \text { Transmission crystal spectrometer }\end{array}$

\section{References}

1. M. A. Duguay and P. M. Rentzepis, "Some approaches to vacuum UV and X-ray lasers," Appl. Phys. Lett. 10, 350 (1967).

2. A. V. Vinogradov and I. I. Sobel'man, "The problem of laser radiation sources in the far ultraviolet and X-ray regions," Sov. Phys. JETP 36, 1115 (1973).

3. G. Chapline and L. Wood, "X-Ray Lasers," Phys. Today, pp. 40-48 (june 1975).

4. A. V. Vinogradov, I. I. Sobelman, and E. A. Yukov, "Possibility of constructing a farultraviolet laser utilizing transitions in multiply charged ions in an inhomogeneous plasma," Sov. J. Quantum Electron. 5, 59 (1975). 
5. A. N. Zherikhin, K. N. Koshelev, and V.S. Letokhov, "Gain in the far vacuum ultraviolet region due to transitions in multiply charged ions," Sov. J. Quantum Electron. G, 82 (1976).

6. A. V. Vinogradov, I. I. Sobelman, and E. A. Yukov, "Population inversion of transitions in neon-like ions," Sov. J. Quantum Electron. 7, 32 (1977).

7. A. A. Ilyukhin, G. V. Peregudov, E. N. Ragozin, I. I. Sobel'man, and V. A. Chirkov, "Concerning the problem of lasers for the far ultraviolet," IETP' Lett. 25, 535 (1977).

8. P. O. Egan and J. K. Wobser, Transmission Crystai Spectrometer for Measuring Hard X-Rays Underground, Lawrence I.:vermore National Laboratory, Livermore, CA, UCRL-53929-89, pp. 163-166 (1989).

9. P.T. Springer, Measuring Electron Temperature in Nuclear-Driven Plasmas, Lawrence Livermore National Laboratory, Livermore, CA, UCRL-53929-89, pp. 13-19 (1989).

10. R. E. Stewart, M. W. Bowers, M. R. Carter, and D. F. Price, Time-Resolving Intensified Detectors for Low-Level X-Ray Detection, Lawrence Livermore National Laboratory, Livermore, CA, UCRL-53929-88, pp. 60-63 (1988).

1. D. L. Matthews, P. L. Hagelstein, M. D. Rosen, M. J. Eckart, N. M. Ceglio, A. U. Hazi, H. Medecki, B. J. MacGowan, J. E. Trebes, B. L. Whitten, E. M. Campbell, C. W. Hatcher, A. M. Hawryluk, R. L. Kauffman, L. D. Pleasance, G. Rambach, J. H. Scofield, G. Stone, and T. A. Weaver, "Demonstration of a Soft X-Ray Amplifier," Phys. Rev. Lett. 54, 110 (1985).

12. M. D. Rosen, P. L. Hagelstein, D. L. Matthews, E. M. Campbell, A. U. Hazi, B. L. Whitten, B. MacGowan, R. E. Turner, and R. W. Lee, "Exploding-Foil Technique for Achieving a Soft X-Ray Laser," Phys. Rev. Lett. 54, 106 (1985).

13. B. J. MacGowan, L. B. DaSilva, D. J. Fields, C. J. Keane, J. A. Koch, R. A. London, D. L. Matthews, S. Maxon, S. Mrowka, A. L. Osterheld, J. H. Scofield, G. Shimkaveg, J. E. Trebes, and R.S. Walling, "Short wavelength $\mathrm{X}$-ray laser research at the Lawrence Livermore National Laboratory," Phys. Fluids B 4, 2326 (1992).

14. L. B. DaSilva, J. E. Trebes, S. Mrowka, T. W. Barbee, Jr., J. Brase, J. A. Koch, R. A. London, B. J. MacGowan, D. L. Matthews, D. Minyard, G. Stone, T. Yorkey, E. Anderson, D. T. Attwood, and D. Kern, "Demonstration of $\mathrm{x}$-ray microscopy with an $\mathrm{x}$-ray laser operating near the carbon K edge," Opt. Lett. 17, 754 (1992).

15. L. B. DaSilva, J. E. Trebes, R. Balhorn, S. Mrowka, E. Anderson, D. T. Attwood, T. W. Barbee, Jr., J. Brase, M. Corzett, J. Gray, J. A. Koch, C. Lee, D. Kern, R. A. London, B. J. MacGowan, D. L. Matthews, and G. Stone, "X-ray Laser Microscopy of Rat Sperm Nuclei," Science 258, 269 (1992).

16. N. M. Ceglio, D. G. Stearns, D. P. Gaines, A. M. Hawryluk, and J. E. Trebes, "Multipass amplification of soft $X$-rays in a laser cavity," Opt. Lett. 13, 108 (1988).

17. N. M. Ceglio, D. P. Gaines, J. E. Trebes, R. A. London, and D. G. Stearns, "Time-resolved measurement of double-pass amplification of soft X-rays, Appl. Opt. 27, 5022 (1988).

18. R. M. Bionta, E. Ables, O. Clamp, O. D. Edwards, P. C. Gabriele, K. Miller, L. L. Ott, K. M. Skulina, R. Tilley, and T. Viada, "Tabletop X-ray microscope using $8 \mathrm{keV}$ zone plates," Opt. Engineering 29, 576 (1990).

19. P. L. Hagelstein and R. K. Jung, "Relativistic distorted-wave calculations of electron collision cross sections and rate coefficients for Ne-like ions," Atomic Data and Nuclear Data Tables 37, 121 (1987).

20. I. P. Grant, B. J. McKenzie, P. H. Norrington, D. F. Mayers, and N. C. Pyper, "An a'omic multiconfigurational Dirac-Fock pa:kage," Comput. Phys. Comm. 21, 207 (1980).

21. M. Klapisch, "A program for atomic wavefunction computations by the parametric potential method," Comput. Phys. Comm. 2, 239 (1971).

22. M. Klapisch, J. L. Schwob, B. S. Fraenkel, and J. Oreg, "The $1 \mathrm{~s}-3 \mathrm{p} \mathrm{K} \beta$-like X-ray spectrum of highly ionized iron," J. Opt. Soc. Am. 67, 148 (1977).

23. A. Bar-Shalom, M. Klapisch, and J. Oreg, "Electron collision excitations in complex spectra of ionized heavy atoms," Phys. Rev. A 38, 1773 (1988).

24. J. Nilsen, "Radiative-Hydro Modeling and Atomic Data Bases," AIP Conference Proceedings 168 - Atomic Processes in Plasmas, pp. 5158, A. Hauer and A. L. Merts Eds., American Institute of Physics, New York (1988).

25. D. C. Eder and H. A. Scott, "The calculation of line transfer in expanding media," I. Quant. Spectros. Radiat. Transfer 45, 189 (1991).

26. D. Griswold, R. Mayle, and S. Maxon, "Simulations of a non-uniform line focus on a 
Ni-like Ta x-ray laser," Bull. Am. Phys. Soc. 37, 1459 (1992).

27. T. M. Tillotson and L. W. Hrubesh, "Transparent ultralow-density silica aerogels prepared by a two-step sol-gel process," J. Non-Cryst. Solids 145, 44 (1992).

28. L. W. Hrubesh, "Aerogels: The world's lightest solids," Chemistry \& Industry, p. 824 (December 17, 1990).

29. L. W. Hrubesh and R. W. Pekala, "Frozen Smoke and Other Insubstantials: The Development of Aerogels at LLNL," Energy \& Technology Review, Lawrence Livermore National Laboratory, Livermore, CA, p. 20 (January 1991).

30. R. L. Morrison, "The Versatile Biofoam Solid," The World \& I, p. 244 (November 1992).

31. C. M. Logan, "Digital Mammography," Energy \& Technology Review, Lawrence Livermore National Laboratory, Livermore, CA pp. 27-36 (October-November-December 1992).

32. J. Weisman, "Labs' find may save heart patients," Tri-Valley Herald, Alameda Newspaper Group, Pleasanton, CA, p. B-4 (November 15, 1992).

33. J. H. Kinney and M. C. Nichols, "X-ray tomographic microscopy (XTM) using synchrotron radiation," Annu. Rev. Mater. Sci. 22, 121 (1992).
34. D. L. Lewis, C. M. Logan, and M. R. Gabel, "Continuum $x$-ray gauging of multipleelement materials," Spectroscopy 7, 40 (1992).

35. M. A. Levine, R. E. Marrs, J. R. Henderson, D. A. Knapp, and M. B. Schneider, "The Electron Beam Ion Trap: A New Instrument for Atomic Physics Measurements," Physica Scripta T 22, 157 (1988).

36. R. E. Marrs, M. A. Levine, D. A. Knapp, and J. R. Henderson, "Measurement of electronimpact-excitation cross sections for very highly charged ions," Phys. Rev. Lett. 60, 1715 (1988).

37. D. R. DeWitt, D. Schneider, M. H. Chen, M. W. Clark, J. W. McDonald, and M. B. Schneider, "Dielectronic Recombination Cross Sections of Neonlike Xenon," Phys. Rev. Lett. 68, 1694 (1992).

38. P. Beiersdorfer, J. Nilsen, A. Osterheld, D. Vogel, K. Wong, R. E. Marrs, and R. Zasadzinski, "Measurements of line overlap for resonant photopumping of $4 \rightarrow 4$ transitions in highly charged nickel-like ions," Phys. Rev. A 46, 25 (1992).

39. J. Nilsen, P. Beiersdorfer, S. R. Elliott, and A. L. Osterheld, "An argon-pumped yttrium x-ray laser," Physica Scripta 47, 42 (1993).

40. P. Beiersdorfer, A. L. Osterheld, J. Scofield, B. Wargelin, and R. E. Marrs, "Observation of Magnetic Octupole Decay in Atomic Spectra," Phys. Rev. Lett. 67, 2272 (1991). 

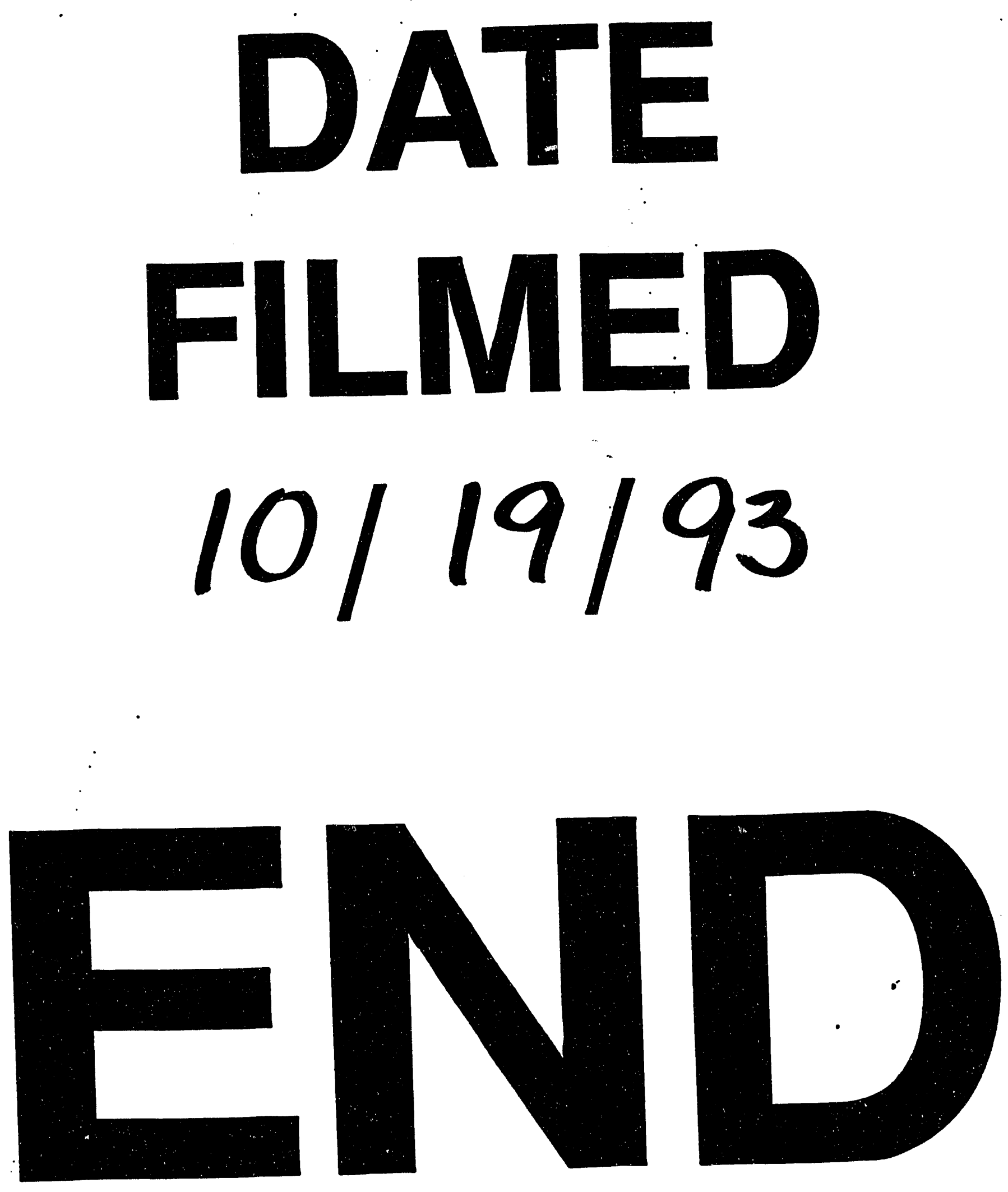
